\title{
Erratum: Saliency-Guided Consistent Color Harmonization
}

Yoann Baveye, Fabrice Urban, Christel Chamaret, Vincent Demoulin, and Pierre Hellier

Technicolor Research and Innovation, Rennes, France

\{baveyey, urbanf, chamaretc, demoulinv, hellierp\} @technicolor.com

https://research.technicolor.com/rennes/

S. Tominaga, R. Schettini, and A. Trémeau (Eds.): CCIW 2013, LNCS 7786, pp. 105-118, 2013.

(C) Springer-Verlag Berlin Heidelberg 2013

\section{DOI 10.1007/978-3-642-36700-7_26}

In the original version, reference 16 was wrong. It should read as follows:

16. Skurowski, P., Kozielski, M.: Investigating human color harmony preferences using unsupervised machine learning. In: European Conference on Colour in Graphics, Imaging, and Vision, pp. 59-64 (2012) 